\title{
Cylindrical Medicine Bottle's Character Recognition of Automotive Dispensing Machine
}

\author{
Mingde Gong, Xianming Zhang* and Tao Ni \\ School of Mechanical Science and Engineering, Jilin University, Changchun city, China \\ ${ }^{*}$ Corresponding author
}

\begin{abstract}
-a new scheme was designed to recognize the medicine which was packed in small cylindrical bottle with a label on the surface. To recognize the label of medicine a new algorithm was needed, which was used to rectify the cylindrical character to plane one. According to the equations of the rectification algorithm, some related positons have to be design in a special structure. After having rectified the label, the technique of OCR or the algorithm SIFT (David Lowe) was referred to recognize the certain character of the medicine label. This scheme could use to recognize and sort the medicine in the automatic dispensing machine.
\end{abstract}

Keywords-cylindrical; medicine; National Medicine Permit No.; recognition; OCR

\section{INTRODUCTION}

In some large scale hospital, there are numerous injections to be made. Generally, the preparation of the present stage of our country was completed by medical staff in injection room or ward therapeutic room. The intensity of work and low efficiency of preparation are the disadvantages[1]. To make up these disadvantages, some automatic dispensing machine had been invented.

Normally, when doing the dispensation, the medical stuff was asked to check the medical prescribe, which must be executed without any mistake. Also in the medicine dispensing machine the medicine checking job has to be done automatically by the medicine recognition system.

There were three methods of medicine recognition. One was using bar code, in this method a new label containing bar code should be attached to the bottle. When doing the recognition, a reader was needed to scan. With this technique, efficiency of drug administration had been greatly improved, manpower saved, data errors reduced[2]. While a fatal weakness was that there hasn't unified bar code for drugs(BCD)[3]. This surely can't be applied universally.

Another way was the radio-frequency technique. RFID is an automatic untouched recognition technique, it recognizes and collects relative data of objects by sending radio-frequency signal[4][5]. In this project, a RF-ID, an aerial and a reader are necessary[6]. The success rate can nearly reach $100 \%$, which however was definitely sophisticated and high cost.

Thirdly, optical recognition was also developed fast and many merits had been achieved. The Ying Li[7] has done the job by comparing the label figure taken in real time with the standard one stored before. Calculating the difference of the two figures, only if the value belongs to the special range which was defined as the criteria of success recognition, can get the right result. This algorithm ignored the direction of the label, which was obvious too difficult to fulfill that, causing the difference in theory.

So as far, there were few work has been done with Cylindrical Character Recognition. It's necessary to develop new theory to fulfill the vacancy.

\section{Build THE STRUCTURE OF THE SCHEME}

Before inducting the algorithm, a new structure of the system should be made and understood. As an equipment that would be used in automatic dispensing machine, the medicine bottle has to be moved continuously passing the recognition zone and reaching to the dispensing zone. So the bottle has to be carried by a conveyer belt. When putted on the belt, the label should face up to the camera located along the belt in crossing direction. Behind the camera was a sorting equipment (used to kick out the wrong medicine, but not the object that would be introduced). The Figure I is the illustrative diagram.

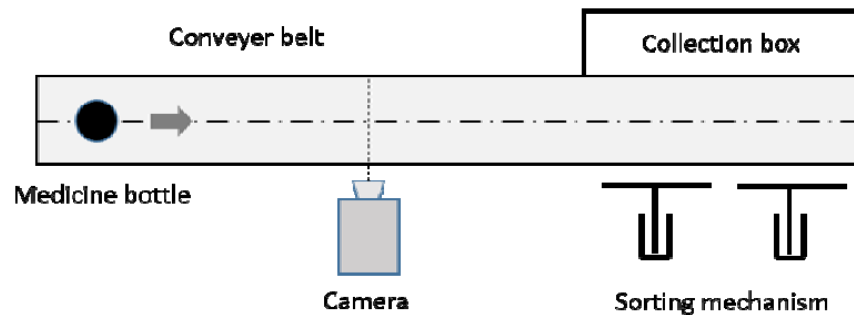

FIGURE I. THE STRUCTURE OF THE SCHEME

When the medicine bottle moving through the camera zone, an image will be taken. This image should contain cylindrical character, including a distorted label figure. By making an rectification operation, the characters of National Medicine Permit No. can be recognized with OCR and SIFT algorithm, getting the medicine recognized by the way.

\section{CHARACTER RECTIFICATION AlgORITHM}

When bottle passing through the camera, an image was taken immediately. But the characters in image could not be recognized directly for having been distorted, especially near the bottle edge, as was shown in the figure II. According to pinhole imaging principle, a cylinder surface character can be 
rectified with mathematical equations with a precondition of exact geometry dimensions.

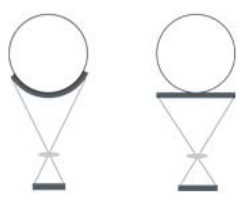

FIGURE II. THE DISTORTION DIAGRAM

The basic principle doing the image distortion was that cutting the image by $\mathrm{N} \times 1, \mathrm{~N}$ equals to the column dimension of the image and then extend the cut figure wider by multiple a corresponding ratio as the core factor to rectify the image, to make the rectified figure unit(n) with the dimension of $\mathrm{N} \times$ ratio.

The figure III is the illustrative diagram of the undistortion. In the equotions calculating the ratios, a serial triangle similar was refered. Then the arc $\mathrm{AB}$ and segment $\mathrm{CD}$ was measured, and the ratio $=\mathrm{AB} / \mathrm{CD}$. To lay out the equotions, it's necessary to define some various: $\mathrm{L}$ represent the distance between the center of bottle and the focus of the camera. The distance $d$ means the focal length. The letter $\mathrm{n}$ and $\mathrm{s}$ respectively means the number of the pxiel and the demension of single pixel. Take the No. n column pixels as example, the derivation of formula are developed as follows:

$$
\begin{aligned}
& \sin \alpha=\frac{n}{\sqrt{\ln s} x^{2}+d^{2}} \\
& \cos \alpha=\frac{d}{\sqrt{\ln s x^{2}+d^{2}}} \\
& \cos \beta=\frac{(a+1) g}{\sqrt[3]{(n g}(n)+1)^{2}+\alpha^{2}} \\
& \sin \beta=\frac{\alpha}{\sqrt{3(n+1) g^{2}+d^{2}}} \\
& \cos \Psi=\frac{\left(\operatorname{Lsin}^{2}\right)^{2}+\operatorname{sosm} \sqrt{B^{2}-(\operatorname{Linm})^{2}}}{\mathrm{~B} .} \\
& \cos \theta=\frac{\left(\operatorname{Lin} \beta^{2}\right)^{2}+\operatorname{cosen} \sqrt{B^{2}-(\operatorname{Lin} \beta)^{2}}}{\mathrm{~B}}
\end{aligned}
$$

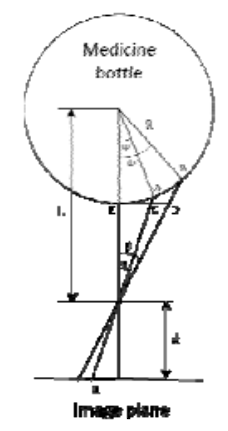

FIGURE III. THE ILLUSTRATIVE DIAGRAM OF EQUATIONS

$$
\begin{gathered}
\operatorname{arc}=R\left(\cos \phi^{-1}-\cos \Psi^{-1}\right) \\
\text { ratlo }=\frac{\operatorname{arc} \cdot d}{\left(\left[-\operatorname{Rig}_{2}\right.\right.}
\end{gathered}
$$

Each parameters must be exact which can be restrianed by some physical factors. If there exist any noteble mistake, ether the undistorted image would not be rectified properly or some ratios belong to imaginary number. We supposely take each undistorted image as unit(n), the final rectified image.

$$
\text { IMAGE }=\Sigma \text { unit(n) }
$$

Before doing the test, the orignial image has to be operated with filering and binaried. After having drawed an experiment, the correctness had been verified.

The figure IV was the result of the rectification experiment. It's obvious that the distorted edge has been rectified and looked close to the natural plane label.

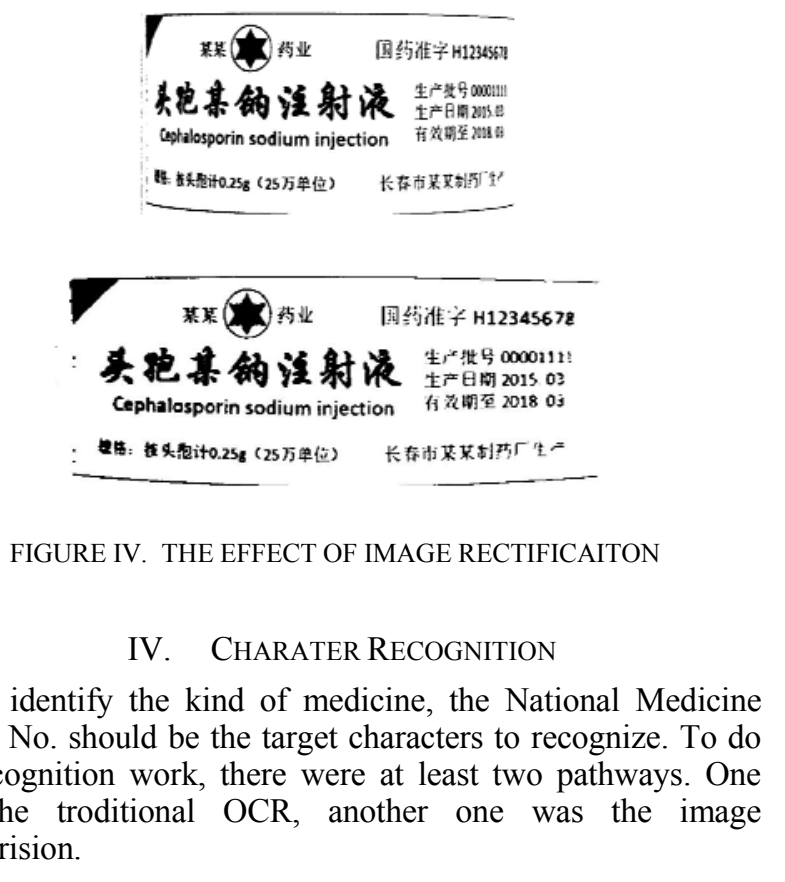




\section{A. Recognize with OCR}

Before using OCR recognition, we have to create a charater data base including all the possible characters of National Medicine Permit No.. The data base was created based on the statistical characteristics[8].

Actually, there were many characters that can distracted, such as the structual characters and other advanced statistical characteristics, as was illustrated in the reference 4. Howerer the simplest statistical characters had been taken, because a simple and reliable recognition rule, has been introduced, which is that when doing the recongnition job comparing each char with that of National Medicine Permit No. in order of from left to right and each match rate has to be noless than $60 \%$. In this conditon we take it as granted that the medicine is exact. base.

Now take “药” as example to display how to creat data

1 st, get the minimum bounding rectangle of the char “药”.

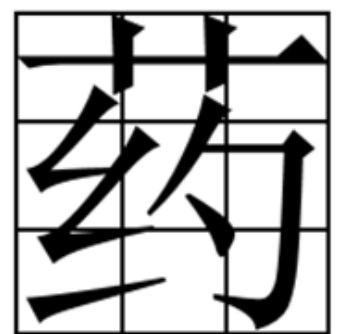

FIGURE V. SEGMENTATION DIAGRAM OF THE A CHAR

$2 \mathrm{nd}$, divid the rectangle into $3 \times 3$ grids, in each part, and do the proportion statistics of 0 pixels. Store these character data into a base used to be refered when doing the recognition. By this method, a charater data base was created and stored.

Before doing the recongnition, some image preproceesing work should be prepared properly. Delecting the uncharacter comes the first. In this section several steps would be taken. Firstly, count the size of each contiuous section of 0 pixels and then set an reasional range. All the information were shown from figure VI to fiugre VIII. Those out of the range should be assigned as uncharacter and repaced by 1 pixels.

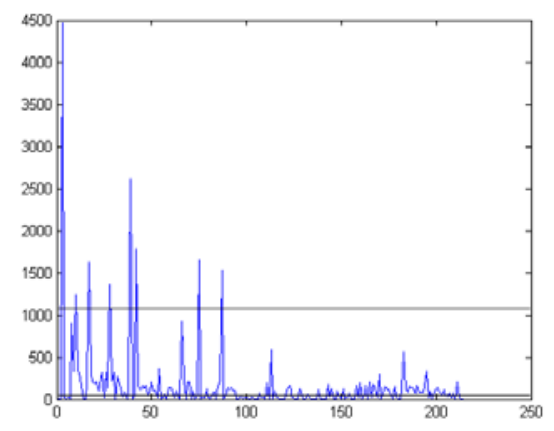

FIGURE VI. THE CONNECTED DOMAIN AREA OF 0 PIXELS

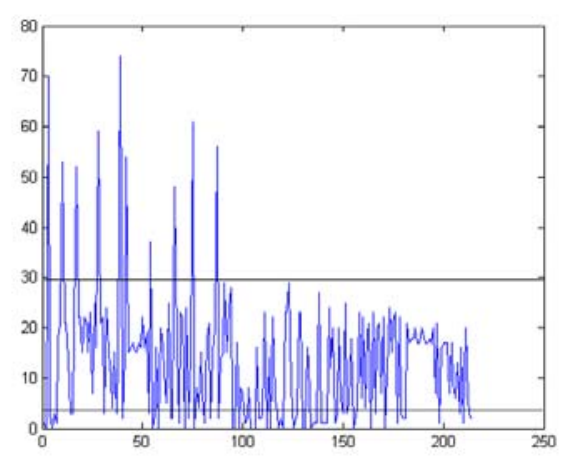

FIGURE VII. THE HIGHT OF CONNECTED DOMAIN OF 0 PIXELS

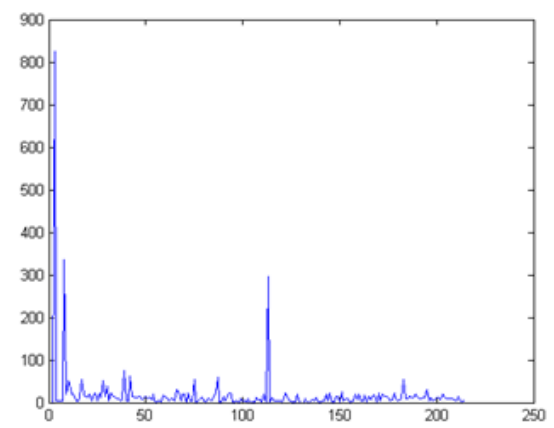

FIGURE VIII. THE WIDTH OF CONNECTED DOMAIN OF 0 PIXELS

Having delected uncharacers, the image was clean, displayed in the figure IX.
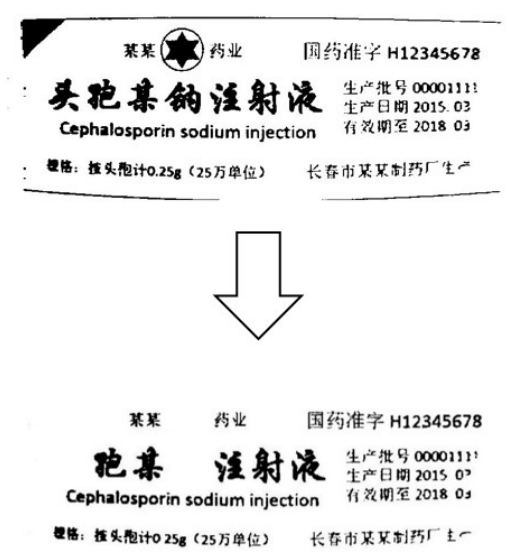

FIGURE IX. THE RESULT OF HAVING DELECTED THE NONCHARACTERS

Secondly, intergrate the similar words must by enlarging the minimum bounding rectangles of each characters and combining them together, forming several indpendent parts like figure $\mathrm{X}$. Each part will be checked one by one. 


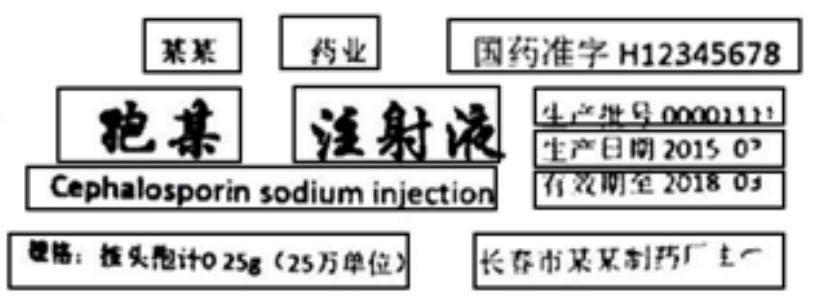
FIGURE X. RELATIVE CHARACTERS THAT SHOULD BE
INTEGRATED

We can take several steps to do the recongnition. 1st, count the quantity of characters in each independent part, and only those equal to 13 can possible be the National Medicine Permit No.(NMPN), regarding “国药准字 H12345678” as recognition critera. $2 \mathrm{nd}$, compare the characters in the remaining part with the data base with same order of that in NMPN and only if ecah match ratio no less than $60 \%$, can we suppose that the medicine was right. By doing the experiment, this method has been verified.

\section{B. SIFT}

Another method was to quote the SIFT algorithm, with main princple to get the keypoints in many layers of scale space[9]. The SIFT algorithm has the rotation invariant property, which can tolerance light noise. By comaring the similarity of keypoints, two similar objects can be matched[10]. To use this method to recognize the medicine, there are mainly two-step work to execute. 1 st, prepare a standard image taken by the camera with an flat medicine label, containing merely the NMPN with blank background. 2 nd, define the limit of number of keypoints matched success. Finaly, do the matching work and get the recognition result displayed in figure XI and figure XII.

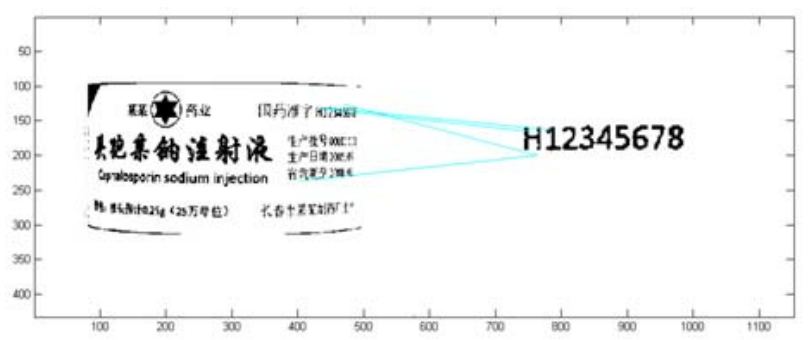

FIGURE XI. RESULT OF HAVING DONE THE MATCH BETWEEN DISTORTED AND STANDARD IMAGE

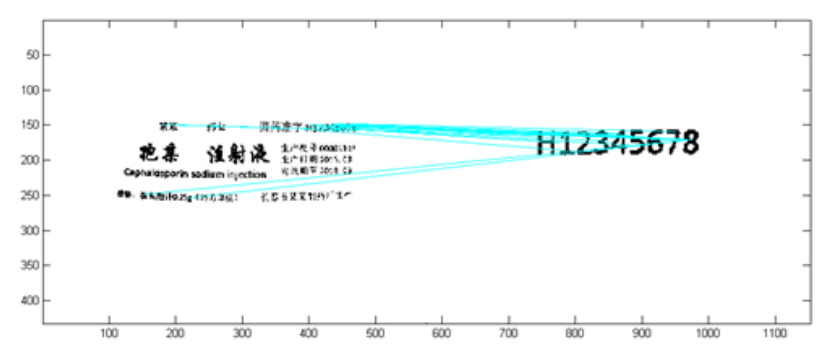

FIGURE XII. RESULT OF HAVING DONE THE MATCH BETWEEN RECTIFICATION AND STANDARD IMAGE
There severally 3 and 27 keypoints were matched seperatlly, with the principle that the more matched points the much similar between the images.

From the result, we can find that the image after doing rectification had got more matched keypoints, certainly much higher match rate, which has verified that the rectification alogrithm was effective and applicable.

\section{CONCLUSION}

To recognize the medicine packed with small cylindrical bottle opticaly, only few work has been conducted. The maxmum difficulty was that the characters in image was not displaied naturely. By introducing an algorithm of rectifying the image, it has provide the precondition of the recognition of the cylindrical character. Both OCR and SIFT algorithm can do the matching work exactly. What was the most meaningful was that it can be used to check out the wrong medicine in the automatic dispensing machine, which delaberate the work and maintain the accuracy, which can do lots of contribution to the healthcare section.

\section{ACKNOWLEGEMENT}

The authors are grateful for partial support from The National Research Foundation for the Doctoral Program of Higher Education of China under Grant No. 20130061110009 and National Natural Science Foundation of China, with the No. of 51305153, and thank professor Mingde Gong and Professor Tao Ni for the support in the academic guidance.

\section{REFERENCES}

[1] Xueqi Fu, "Design of The Tobot Used for Intravenous Medication Preparation and Control Based on Expert System," Harbin Institute of Technology. 2015.

[2] Hongbin Yang, Lin Xiang, Li Chen, Wen Li, “Application of 2D code and PDA in hospital drug management," Chinese Journal of Hospital Pharmacy 35.23(2015):2142-2145.

[3] Yongfu Peng, Peiyuan Xia, Songqing Liu "The Design of Unified Bar Code for Drugs in China," Western Pharmaceutical Forum of China. 2004:439-441.

[4] Weimin Lang, "Principle and Application of Radio Frequency Identification Technology," China Machine PRESS, 2006.

[5] Zhanqing You, "Planning and Implementation of Radio Frequency Identification Technology," Electronic Industry Press. 2005.

[6] Junfeng Tian, Maohua Zhu, Dazhang Zeng," Research on The Application of Wireless Radio Frequency Identification Technology in Drug Safety Management [J]”. E-Business Journal, 2007(5):68-71.

[7] Ying Li, "A Study on the Method of Checking for Label of Medicine Bottle," Shenyang University of Technology, 2015.

[8] Ye Zhang, Bo Chen. "The principle of character recognition[J]," China Equipment, 2009(12).

[9] David G. Lowe, "Distinctive image features from scale-invariant keypoints," International Journal of Computer Vision, 60, 2 (2004), pp. 91-110.

[10] David G. Lowe, "Object recognition from local scale-invariant features," International Conference on Computer Vision, Corfu, Greece (September 1999), pp. 1150-1157. 